\title{
Control permanente de la gestión sanitaria por monitoreo de costos mediante acontecimientos indicadores
}

\author{
Horacio Villanueva ${ }^{1}$
}

RESUMEN Objetivos. Determinar el costo operacional de un servicio quirúrgico, comparar los resultados con estándares y determinar la incidencia de costos. Obtener una metodología eficaz para este fin, extrapolable a todo tipo de servicio asistencial y de fácil instrumentación en una herramienta informática convencional (base de datos) de alta difusión y bajo costo que pueda usarse en el control permanente de la gestión.

Métodos. El estudio se realizó durante 1999 en el Servicio de Ortopedia y Traumatología del Hospital Estatal "Domingo Funes", Córdoba, Argentina. De los 817 pacientes sometidos a procedimientos diagnósticos o terapéuticos en ese servicio a lo largo del año, se seleccionaron los 154 que necesitaron internación y maniobras quirúrgicas o instrumentales. Se utilizó el método de acontecimientos indicadores descrito por Kessner. Dichos acontecimientos, clasificados en función de su complejidad (alta, mediana, escasa o nula), fueron seleccionados y definidos por consenso de expertos: especialistas médicos y economistas. Se valoraron los costos directos e indirectos para estudiar su peso en el costo total, en cuya estimación se utilizaron nueve variables que se aplicaron a los diferentes acontecimientos indicadores.

Resultados. El mayor peso del costo total, tanto estándar como real, recayó en ambos casos sobre la variable "día cama". De la variable "día cama" en el total de acontecimientos indicadores, el 51,2\% correspondió a la internación social (costo indirecto) y el 48,8\% a la internación terapéutica (costo directo). Su diferencia con respecto al costo estándar fue un aumento del orden del $117 \%$. Los costos indirectos (internación social e insumos terapéuticos) absorbieron casi la mitad del costo real total. El costo real de todos los acontecimientos investigados, sin incluir los costos indirectos, solo superó al costo estándar en un 6,4\%. Incluyendo los costos indirectos (internación social e insumos terapéuticos) el costo real superó al costo estándar en un 39,8\%. El total gastado en recursos humanos médicos y auxiliares solo representó un $11,5 \%$ del costo total.

Discusión. Se hacen sugerencias con el fin de aportar soluciones a problemas como el alto costo de la internación social y los insumos terapéuticos, los beneficios de la protocolización de procedimientos y tratamientos en el control de costos, la utilización racional de la tecnología para mejorar la eficiencia con disminución de riesgos, etc. Asimismo, se recomienda la extrapolación de este procedimiento para el control de la gestión de costos en todo servicio en donde se realicen procedimientos quirúrgicos o médicos.

Palabras clave Gestión sanitaria, costos directos, costos indirectos.

1 Escuela de Salud Pública. Universidad Nacional de Córdoba. Hospital Estatal Domingo Funes, Córdoba, Argentina. Toda la correspondencia debe ser enviada a Horacio Villanueva a la siguiente dirección: Ayacucho 139, Piso 2, Oficina 6. CP 5000. Córdoba, Argentina. Dirección electrónica: villanueva @powernet.net.ar
Los estudios sobre la eficiencia de los servicios de salud se basan en la determinación de los costos económicos con el fin de suministrar la mejor atención médica y obtener el mayor mejo- ramiento posible de la salud de la comunidad, esto es, el mayor beneficio con la menor cantidad de recursos financieros. Sin embargo, como expresa Donabedian $(1,2)$, la eficiencia en ma- 
teria de salud tiene otra dimensión esencial además de la económica, la ética, que hace que un procedimiento no pueda ser elegido solamente por ser menos costoso, sino por su fin último, que es la salud humana (3). De aquí nace el concepto de calidad de los servicios (4). Para lograr este objetivo es necesario obtener el mayor beneficio de los costos, utilizando los recursos con amplitud de criterio, en contraposición a la concepción contable del costo, que consiste en el asentamiento de los egresos de caja por unidad de prestación. Un ejemplo de utilización eficiente de los recursos es el del paciente que necesita rehabilitación tras una intervención quirúrgica. Su realización en el hospital prolonga la internación y aumenta el costo, pero no mejora la calidad de los resultados. En cambio, su realización en el domicilio del enfermo proporciona los mismos resultados y es menos onerosa, con lo que se obtiene una mayor eficiencia.

En los hospitales estatales argentinos, con la sola excepción de algunos autogestionados de la Capital Federal, lo que siempre se ha evaluado durante años es la eficacia, pero nunca se han realizado estudios de costos para determinar la eficiencia de las prestaciones (5-7). Sin embargo, el crecimiento actual del costo en la producción de servicios de salud necesita respuestas apropiadas por parte de los planificadores de las políticas de salud y de los gerentes que las ejecutan (8-10). Para ello es necesario identificar los costos de producción mediante el estudio del costo monetario de los insumos, de las funciones y de los procesos de producción (en donde se encuadran una gran cantidad de variables observables y mensurables), no como gasto meramente contable, sino como concepto económico de costo. De esta manera se puede individualizar la incidencia de cada una de las variables, identificar su peso proporcional en el costo final y actuar en consecuencia mediante acciones gerenciales y de control de la gestión.

Existen otros estudios de costos con enfoque global epidemiológico (11), pero el propósito general de este trabajo fue sentar las bases de un sistema de información versátil que valore no solo el gasto contable, sino que permita medir los valores económicos del costo y sea aplicable al control de gestión de todos los servicios quirúrgicos o médicos con internación. Sus objetivos específicos consistieron en: 1) determinar el costo operacional real del Servicio de Ortopedia y Traumatología de un hospital estatal argentino; 2) compararlo con el costo estándar para su ajuste permanente; 3) determinar la incidencia del costo indirecto en el costo final; 4) obtener un método eficaz para el estudio de costos en un servicio quirúrgico; 5) explorar la posibilidad de aplicarlo en otros servicios, y 6) diseñar una herramienta informática que simplifique la tarea de recolección de datos y su valoración.

\section{MATERIALES Y MÉTODOS}

El estudio se realizó durante 1999 en un servicio quirúrgico de alta complejidad: el Servicio de Ortopedia y Traumatología del Hospital Estatal "Domingo Funes", de la provincia de Córdoba, Argentina. Durante ese año se atendió a 817 pacientes que fueron sometidos a procedimientos diagnósticos o terapéuticos. Para este estudio se seleccionaron solo los procedimientos terapéuticos que necesitaron internación y maniobras quirúrgicas o instrumentales $(n=154)$, por ser los de mayor repercusión en los costos de la atención ( $85 \%$ del costo anual total del servicio).

\section{Identificación de acontecimientos indicadores y variables}

Se utilizó el método de acontecimientos indicadores (AI) descrito por Kessner (12) y, partiendo de las experiencias en investigación de otros autores (13-15), se sentaron las bases de un método de control de gestión de costos, que mediante la observación de una muestra significativa de un conjunto de tratamientos paradigmáticos, agrupados como AI de una misma complejidad, permitiera tener una visión integral del gasto en un servicio de ortopedia y traumatología. Los tratamientos indicadores seleccionados cumplieron al menos cuatro de los siguientes requisitos para ser elegidos como paradigmáticos (16) de los costos y eficiencia de la atención traumatológica y ortopédica:

- Que la tasa de prevalencia fuera suficientemente elevada como para recopilar datos en todo tipo de población.

- Que existiera una definición clara y el diagnóstico fuera fácil.

- Que el análisis del acontecimiento permitiera una visión completa del costo de todos sus componentes.

- Que la atención médica modificara de forma evidente la evolución y el pronóstico de la enfermedad.

- Que la prestación de determinado servicio tuviera una acción directa sobre las condiciones de salud.

Una vez identificados los tratamientos indicadores, mediante la técnica del consenso de expertos (jefes de servicio de varios hospitales, asociaciones científicas y profesionales de la economía vinculados al sector de la salud), se definieron cuatro tipos de AI (cuadro 1), clasificados en función de su complejidad - alta (AIAC), mediana (AIMC), escasa (AIEC) o nula (AINC) - de acuerdo con los siguientes criterios:

- Internación mayor o menor de dos días.

- Uso de la unidad de cuidados intensivos (UCI) o unidad de terapia intensiva (UTI)

- Uso de radioscopia intraoperatoria con intensificador de imágenes.

- Uso postoperatorio de antibióticos.

- Necesidad de dos o más actos quirúrgicos consecutivos.

Nuevamente mediante consulta con expertos y análisis de 10 casos de cada uno de los AI, se definieron sus estándares en procedimientos, maniobras, internación, medicamentos utilizados, etc., y se les asignaron los costos (17) aportados por el Departamento de Costos del hospital.

Para estimar los costos de cada AI se utilizaron nueve variables, cada una de ellas correspondiente a un ámbito indispensable para los tratamientos se- 
CUADRO 1. Acontecimientos indicadores utilizados

\begin{tabular}{cccc}
\hline $\begin{array}{c}\text { Alta complejidad } \\
(n=32)\end{array}$ & $\begin{array}{c}\text { Mediana complejidad } \\
(n=39)\end{array}$ & $\begin{array}{c}\text { Escasa complejidad } \\
(n=33)\end{array}$ & $\begin{array}{c}\text { Nula complejidad } \\
(n=50)\end{array}$ \\
\hline Prótesis total de la cadera & Osteosíntesis de cadera o fémur & Osteosíntesis no femorales & Yeso miembro superior \\
Fractura expuesta + síntesis & Discectomía lumbar & Tenorrafias & Yeso miembro inferior \\
Fracturas del acetábulo & Pseudoartrosis con injerto & Decorticaciones & Toilette de heridas \\
Artrodesis vertebral cervical & Luxación recidivante del hombro & Hallux valgus & Corsé enyesado \\
Instrumentación vertebral & Prótesis total de rodilla & Meniscectomía & Vendaje tipo Shanz \\
\hline
\end{tabular}

leccionados, y todas fueron desglosadas en su estructura íntima de costos con el fin de hacer más exacta la recopilación de la información (figura 1). Con la sola eliminación de una variable (sala de yesos) y la posible inclusión de alguna más por consenso, se puede aplicar esta misma estructura de investigación al estudio de los costos de otras actividades quirúrgicas.

Se analizaron los costos tanto directos, es decir, generados por el prestador de la atención médica (el hospital), como indirectos, esto es, atribuibles al paciente. Entre los costos indirectos se valoró el costo de los insumos terapéuticos (implantes o prótesis) y de la prolongación de la internación por motivos sociales. Este último solo se calculó para los indicadores reales, ya que es imposible valorarlo como estándar. Este análisis se aplicó a cada AI para hacer comparaciones entre los valores estándar y los reales y determinar la incidencia de los costos directos e indirectos y de cada una de las variables examinadas en el costo total de la atención médica. De esa manera fue posible identificar dónde radica el mayor peso del costo de cada AI y sus desviaciones del estándar. Se calculó el valor absoluto del costo de cada variable y su valor porcentual con respecto al costo total. Los valores absolutos se expresaron en dólares estadounidenses (USD), dada su paridad con el peso argentino.

\section{Definición de variables}

El costo medio estándar de cada AI se calculó teniendo en cuenta la incidencia proporcional de sueldos, insumos, limpieza, electricidad, agua, administración, utilización de espacio, servicios, amortización del edificio, alimentación, ropería, etc. (17). A modo de ejemplo, en el apéndice 1 se presenta la definición del estándar de los AIAC y de una de las nueve variables

FIGURA 1. Árbol de variables

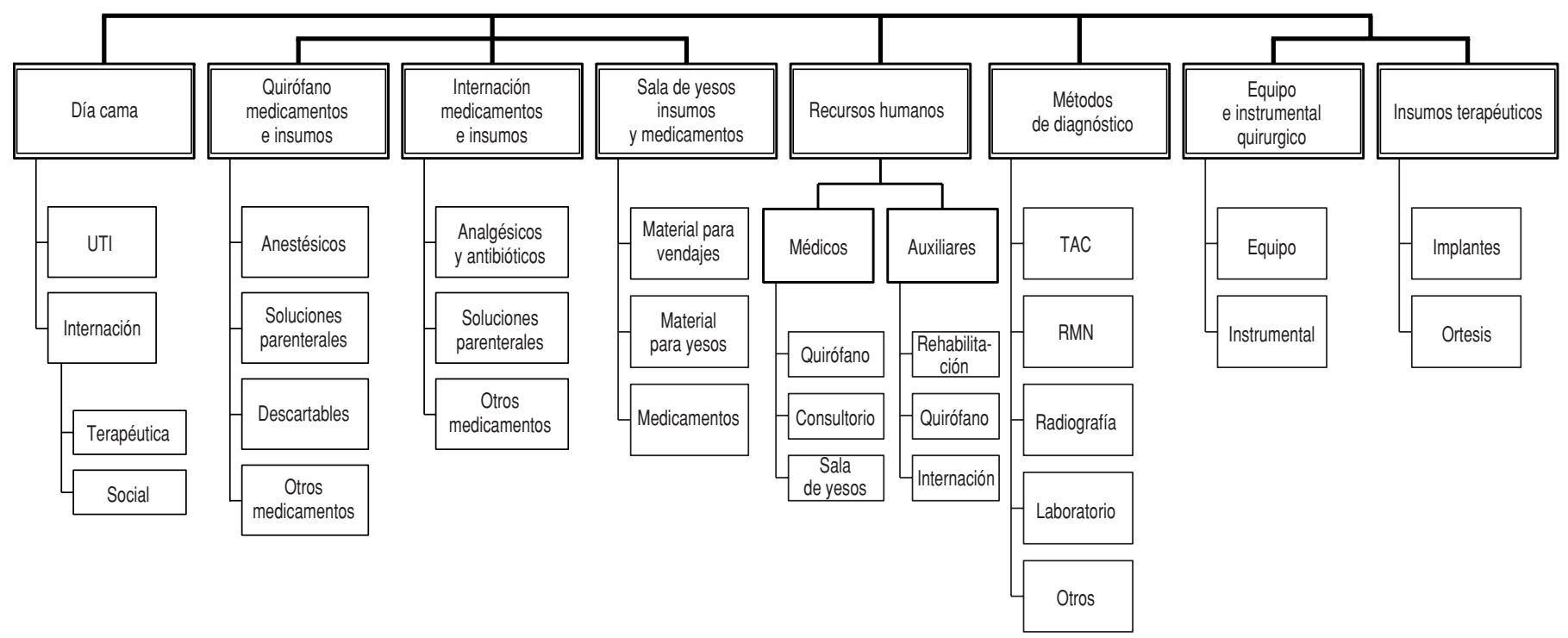


(quirófano: medicamentos e insumos). Una vez calculados los costos reales de cada AI, se compararon con los costos estándar.

\section{RESULTADOS}

Por razones de espacio, solo se realiza un breve comentario sobre el costo total de todos los acontecimientos y se muestran los resultados del análisis general de los AI y los costos comparativos de cada uno de ellos.

La comparación de los costos totales estándar y reales revela, a través de sus porcentajes, que el mayor peso recayó en ambos casos sobre la variable "día cama", que representó un 39\% del costo estándar y un 56\% del costo real (cuadro 2). Los costos indirectos absorbieron casi la mitad del costo real total (46,2\%): internación social, $25,4 \%$, e insumos terapéuticos, $20,8 \%$, frente a un $53,8 \%$ para los costos directos.

Los cuadros 3 a 6 muestran el peso de cada una de las variables, expresadas en porcentajes y en valores absolutos, para cada uno de los tipos de AI: AIAC, AIMC, AIEC y AINC, respectivamente.

El costo estándar de un AIAC se estimó en US\$ 4 323, mientras que el costo real fue de US\$ 6075 (cuadro 3). Del $48,6 \%$ del costo total que representó la variable "día cama", el 27,2\% correspondió a costos indirectos (internación social) y el 21,4\% a costos direc-

CUADRO 2. Comparación de los costos totales estándar y real de los 154 pacientes

\begin{tabular}{|c|c|c|c|c|}
\hline & \multicolumn{2}{|c|}{ Costo estándar } & \multicolumn{2}{|c|}{ Costo real } \\
\hline & $\%^{a}$ & US $\$ b$ & $\%^{a}$ & US $\$ b$ \\
\hline Día cama & 31,0 & 98570 & 49,7 & 213803 \\
\hline Insumos terapéuticos & 31,4 & 99900 & 20,8 & 89299 \\
\hline Quirófanoc & 20,3 & 64416 & 18,4 & 79311 \\
\hline Otros costos & 17,3 & 54863 & 11,1 & 47769 \\
\hline
\end{tabular}

CUADRO 3. Comparación entre los costos estándar y real por paciente de los acontecimientos indicadores de alta complejidad

\begin{tabular}{lrrrrr}
\hline & \multicolumn{2}{c}{ Costo estándar } & & \multicolumn{2}{c}{ Costo real } \\
\cline { 2 - 3 } \cline { 5 - 6 } & $\%^{\mathrm{a}}$ & US\$ & & $\%^{\mathrm{a}}$ & US $\$^{\mathrm{b}}$ \\
\hline Día cama & 35,2 & 1520 & & 48,6 & 2957 \\
Quirófano & 4,9 & 214 & & 3,3 & 203 \\
Internación & 7,8 & 339 & & 4,6 & 281 \\
Sala de yesos & - & - & & 1,2 & 71 \\
Recursos humanos médicos & 7,8 & 338 & & 7,3 & 443 \\
Recursos humanos auxiliares & 4,6 & 198 & & 5,2 & 314 \\
Métodos de diagnóstico & 9,7 & 419 & & 6,1 & 369 \\
Equipo e instrumental & 2,2 & 95 & & 1,5 & 91 \\
Insumos terapéuticos & 27,8 & 1200 & & 22,2 & 1346 \\
$\quad$ Total & 100 & 4323 & & 100 & 6075 \\
\hline
\end{tabular}

a Porcentajes del total.

${ }^{b}$ Dólares estadounidenses.

tos (internación terapéutica). Los costos indirectos de los AIAC (insumos e internación social) representaron el $49,4 \%$ del costo total de esos acontecimientos (cuadro 3).

El costo estándar de un AIMC se estimó en US\$ 2 650, mientras que el costo real fue de US\$ 3 759. Del 46,2\% del costo total que representó la variable "día cama", el 17,9\% correspondió a costos indirectos (internación social) y el $28,3 \%$ a costos directos (internación terapéutica). Las variables "día cama" e "insumos terapéuticos" representaron un $72,4 \%$ del costo total de los AIMC, y sus costos indirectos (insumos e internación social) un 44,1\% (cuadro 4).

El costo estándar de un AIEC se estimó en US\$ 1 386, mientras que el costo real fue de US\$ 1 881. Del 56,8\% del costo total que representó la variable "día cama", el 31,3\% correspondió a costos indirectos (internación social) y el $25,5 \%$ a costos directos (internación terapéutica). Los costos indirectos (insumos e internación social) representaron el 44,1\% del total (cuadro 5).

El costo estándar de un AINC se estimó en US\$407, mientras que el costo real fue de US\$ 553. Del 58,9\% del costo total que representó la variable "día cama", el 39,7\% correspondió a costos indirectos (internación social) y el 19,2\% a costos directos (internación terapéutica). Los costos indirectos (internación social) representaron el 39,7\% del total (cuadro 6).

\section{DISCUSIÓN}

Según los valores estándar determinados por consenso, el mayor peso del costo total (directo e indirecto) correspondió siempre a las variables "día cama" e "insumos terapéuticos". Por lo tanto, la internación fue el elemento más costoso $\mathrm{y}$, al tratarse de un costo directo, con mayores posibilidades de ser modificado por la institución. La siguiente variable de mayor peso en el costo total, "insumos terapéuticos", resulta mucho más difícil de modificar desde la institución, dado que se trata de un costo indirecto $y$, más aún, por 
CUADRO 4. Comparación entre los costos estándar y real por paciente de los acontecimientos indicadores de mediana complejidad

\begin{tabular}{lrrrrr}
\hline & \multicolumn{2}{c}{ Costo estándar } & & \multicolumn{2}{c}{ Costo real } \\
\cline { 2 - 3 } \cline { 5 - 6 } & $\%^{\mathrm{a}}$ & US $\$^{\mathrm{b}}$ & & $\%^{\mathrm{a}}$ & US $\$^{\mathrm{b}}$ \\
\hline Día cama & 27,4 & 726 & & 46,2 & 1736 \\
Quirófano & 5,8 & 154 & & 4,2 & 157 \\
Internación & 9,1 & 242 & & 6,1 & 230 \\
Sala de yesos & 0,3 & 8 & & 0,3 & 11 \\
Recursos humanos médicos & 7 & 186 & & 5,8 & 220 \\
Recursos humanos auxiliares & 2,9 & 77 & & 3,7 & 139 \\
Métodos de diagnóstico & 10,2 & 270 & & 5 & 189 \\
Equipo e instrumental & 3,3 & 87 & & 2,5 & 93 \\
Insumos terapéuticos & 34 & 900 & & 26,2 & 984 \\
$\quad$ Total & 100 & 2650 & & 100 & 3759 \\
\hline
\end{tabular}

a Porcentajes del total.

b Dólares estadounidenses.

CUADRO 5. Comparación entre los costos estándar y real por paciente de los acontecimientos indicadores de escasa complejidad

\begin{tabular}{lrrrrr}
\hline & \multicolumn{2}{c}{ Costo estándar } & & \multicolumn{2}{c}{ Costo real } \\
\cline { 2 - 3 } \cline { 6 - 6 } & $\%^{\mathrm{a}}$ & US $\$^{\mathrm{b}}$ & & $\%^{\mathrm{a}}$ & US $\$^{\mathrm{b}}$ \\
\hline Día cama & 31,4 & 435 & & 56,8 & 1067 \\
Quirófano & 9,2 & 128 & & 7,4 & 137 \\
Internación & 2,8 & 39 & & 2,8 & 51 \\
Sala de yesos & 0,9 & 12 & & 0,9 & 15 \\
Recursos humanos médicos & 9,4 & 130 & & 7,6 & 141 \\
Recursos humanos auxiliares & 3,5 & 48 & & 5,5 & 101 \\
Métodos de diagnóstico & 5,8 & 81 & & 4,1 & 75 \\
Equipo e instrumental & 0,9 & 13 & & 2,1 & 56 \\
Insumos terapéuticos & 36,1 & 500 & & 12,8 & 238 \\
$\quad$ Total & 100 & 1386 & & 100 & 1881 \\
\hline
\end{tabular}

a Porcentajes del total.

b Dólares estadounidenses.

CUADRO 6. Comparación entre los costos estándar y real por paciente de los acontecimientos indicadores de nula complejidad

\begin{tabular}{lrrrrr}
\hline & \multicolumn{2}{c}{ Costo estándar } & & \multicolumn{2}{c}{ Costo real } \\
\cline { 2 - 3 } \cline { 6 - 6 } & $\%^{\mathrm{a}}$ & US $\$^{\mathrm{b}}$ & & $\%^{\mathrm{a}}$ & US $\$^{\mathrm{b}}$ \\
\hline Día cama & 35,6 & 145 & & 58,9 & 326 \\
Quirófano & 18,4 & 75 & & 8,3 & 46 \\
Internación & 2,3 & 9 & & 0,9 & 5 \\
Sala de yesos & 8,8 & 36 & & 7,8 & 43 \\
Recursos humanos médicos & 15,2 & 62 & & 11,4 & 63 \\
Recursos humanos auxiliares & 1,6 & 6 & & 1,1 & 6 \\
Métodos de diagnóstico & 18,2 & 74 & & 4,9 & 27 \\
Equipo e instrumental & - & - & & 6,7 & 37 \\
Insumos terapéuticos & - & - & & - & - \\
$\quad$ Total & $100 \%$ & 407 & & $100 \%$ & 553 \\
\hline
\end{tabular}

a Porcentajes del total.

${ }^{b}$ Dólares estadounidenses. ser un elemento imprescindible para la eficacia del tratamiento.

Con respecto al costo real total, la variable "día cama" fue también la de mayor peso, y la internación social (costo indirecto) representó más de la mitad de la misma. En Argentina y muchos otros países de América este tipo de internación es originado por diversos motivos inherentes a la población que atienden los hospitales estatales, como la distancia del domicilio de los pacientes, la dificultad de acceso por falta de medios de transporte adecuados, los trastornos familiares por falta de espacio y tiempo para dedicar a la atención de los pacientes con impedimentos físicos o las dilaciones en el suministro de los insumos terapéuticos solicitados. Además, en muchas ocasiones este tipo de internación prolongada se debe solo a una antigua tradición vinculada con los históricos hospitales de beneficencia.

$\mathrm{Si}$ al costo generado por la variable "día cama" se le agrega el de "insumos terapéuticos", se alcanza el 70,5\% del total del gasto de la institución para prestar el servicio. A estas variables les siguen en el orden de costos "recursos humanos médicos", con un 7,1\%, y "métodos de diagnóstico", con el 5,3\%. El costo real de esta última variable fue significativamente inferior al estándar, gracias al uso del intensificador de imágenes, que abarata los costos de las radiografías intraoperatorias. Esta reducción sería aun mayor si sobre los valores reales no hubiera actuado la influencia perniciosa de la internación social, que generó costos que deberían ser de seguimiento ambulatorio y no de internación. En cifras absolutas, el gasto en recursos humanos fue un $36 \%$ superior al estándar, especialmente por el efecto de la internación social, que disparó la atención de rehabilitación y de interconsultas por necesidades postergadas (odontología, oftalmología y otras especialidades médicas) no dependientes de la enfermedad principal y que se deberían haber hecho en régimen ambulatorio. Al comparar el costo real total, excluida la internación social, con el costo estándar, en el que no estaba incluido este componente, se obtuvieron cifras 
similares, con un peso también similar de las diferentes variables. Esto confirma que el método del consenso utilizado para establecer los estándares se aproxima bastante a la realidad.

Comparando los costos directos estándar y reales, la eficiencia fue buena, ya que la diferencia fue solo de un $6,4 \%$. Sin embargo, al incluir los costos indirectos se observó un enorme desfase, cercano al $40 \%$ (costo diario extra por internación social de USD 338). Este gasto generado por razones sociales podría incluirse en el costo estándar al considerar al Estado como administrador y garante de la salud (18), ya que es su obligación dar solución a esas necesidades. Esta obligación no quita la responsabilidad de administrar racionalmente recursos que son cada vez más escasos (19-21) y de esta investigación también surgen posibles soluciones para abaratar estos costos.

Los resultados de este estudio indican que el método de los acontecimientos indicadores es eficaz y permite analizar meticulosamente cada uno de los componentes del costo final de la atención. Esta investigación ha permitido también comparar resultados con los estándares y obtener conclusiones para el ajuste permanente de estos. Además hay que tener en cuenta la sencillez del método, la posibilidad de aplicarlo con cualquier base de datos comercializada de gran difusión y bajo costo, y la posibilidad de adaptarlo a cualquier servicio quirúrgico o clínico. El autor pone a disposición de quien lo solicite el método utilizado en el diseño de la base de datos.

La repercusión de la internación social en el costo podría reducirse utilizando medios habituales de mucho menor costo como podrían ser: a) oportunos y eficientes traslados para controles postoperatorios, con medios de transporte simples (vehículos comunes adaptados); b) en comunidades cercanas al hospital o en las grandes ciuda- des, el establecimiento de una red de control postoperatorio y de rehabilitación en el domicilio del paciente, o c) el establecimiento de convenios con los hospitales municipales periféricos o cercanos, para la estancia de pacientes en alta transitoria, a cambio de instalar consultorios periféricos brindados por los servicios del centro de salud de mayor complejidad. Los costos de estas alternativas serían considerablemente inferiores a los de la internación social, especialmente si también se consideran los costos sociales del desarraigo y la marginación por abandono.

Los "insumos terapéuticos" constituyen otro rubro en el que las pérdidas del Estado, y en muchas ocasiones de las obras sociales y aun de los pacientes, se podrían controlar con simples métodos de consenso y desburocratización, como el establecimiento por consenso entre todos los jefes de servicio de los hospitales o grupo de sanatorios o gerenciadoras, de un vademécum actualizable cada 6 meses, para dar cabida a las modificaciones de los implantes y prótesis que se utilizarán en ese período y la realización de un protocolo de habilitación y calificación de proveedores para autorizarlos a suministrar ciertos tipos de insumos, contrarrestando el mero enfoque mercantilista de "vender" a través de un escritorio, sin ningún tipo de respaldo técnico imprescindible para ciertos insumos.

Los costos de medicamentos e insumos en quirófano e internación también se podrían reducir considerablemente utilizando protocolos de tratamiento por consenso. La utilización de equipos como el intensificador de imágenes portátil devuelve con creces su alto costo de compra en costo de tiempo quirúrgico, de anestesia, resultados terapéuticos más eficaces, ahorro de película radiográfica e irradiación secundaria de personal y pacientes.

En resumen:
- El mayor peso del costo lo soporta la variable "día cama", con un $49,7 \%$ del total del gasto anual (un $24,3 \%$ en internación terapéutica y un $25,4 \%$ en internación social). La segunda responsable del costo final es la variable "insumos terapéuticos" con un $20,8 \%$. Al ser este un costo indirecto, es de difícil solución; ya que involucra estamentos externos al hospital y fundamentalmente económicos.

- De la variable "día cama" en el total de $\mathrm{AI}$, el $51,2 \%$ corresponde a la internación social (costo indirecto) y el $48,8 \%$ a la internación terapéutica (costo directo). Su desviación del estándar es del orden del $117 \%$ de incremento. La responsabilidad mayor de este desfase la cargan los AIMC, pero en todos los AI hubo alta incidencia de internación social.

- En la variable "día cama", el costo real en todos los acontecimientos supera al costo estándar establecido por expertos en un $56,6 \%$, que convertido en valor monetario muestra una diferencia de US\$ 123 309, (prácticamente el costo de la internación social durante el período estudiado). La institución tuvo una pérdida diaria por este ítem de US\$ 338 .

- El costo real de todos los eventos investigados, sin incluir los costos indirectos, supera al valor estándar en un $6,4 \%$. Convertido en valor monetario, US\$ 13843 en el período estudiado.

- El costo real de todos los eventos investigados, incluidos los costos indirectos, (internación social e insumos terapéuticos) supera al valor estándar en un $39,8 \%$. Convertido en valor monetario, US\$ 122668 en el período estudiado.

- El total gastado en recursos humanos médicos y auxiliares tiene una incidencia de solo un $11,5 \%$ en el costo total, en cifras absolutas US\$ 49565 anuales. 


\section{REFERENCIAS}

1. Donabedian A. Los espacios de la salud: aspectos fundamentales de la organización de la atención médica. México, D.F.: Fondo de Cultura Económica; 1989.

2. Donabedian A. Prioridades para el progreso en la evaluación y monitoreo de la calidad de la atención. Salud Publica Mex 1993;35:94-97.

3. Gracia Guillén D. Fundamentos de bioética. Madrid: Eudema; 1989.

4. Donahue KT. La calidad de la atención a la salud en los Estados Unidos: nuevas tendencias. Salud Publica Mex 1993;35:288-290.

5. Homgren CT. Contabilidad financiera. New Jersey: Prentice-Hall; 1988.

6. Backer M, Jacobsen L, Ramirez D. Contabilidad de costos. Buenos Aires: McGraw-Hill; 1993.

7. Musgrove P. The economic crisis and its impact on health care in Latin America. Int J Health Serv 1987;17:411-421.

8. Vicente MA, Baroli CA, Cranevali CA, Corroppoli MD, Franco JA, Kent P, et al. Administración aplicada a organizaciones de salud. Buenos Aires: Osmar D. Buyatti; 1997.

9. De Moraes Novaes H. Manual de gerencia de la calidad. Buenos Aires: Universidad Nacional de Buenos Aires; 1996.
10. Albrecht K, Bradford L. La excelencia en el servicio. Bogotá: Legis; 1990.

11. Alan Dever GE. Epidemiología y administración de los servicios de salud. Córdoba, Argentina: Universidad Nacional de Córdoba; 1991.

12. Kessner D, Kalk C, Singer J. Assessing health-the case for tracers. N Engl J Med 1973;288:189-194.

13. Arredondo A, Damián T. Economic costs for the production of health services: from cost of inputs to cost of case-management. Salud $\mathrm{Pu}$ blica Mex 1997; 39:117-124

14. Thomas J, Longo D. Application of severity measurement for hospital quality managements. Hospital and Health Services Administration 1990;35:221-243.

15. Musgrove P. Cost-effectiveness and health sector reform. HROVP Working Paper $\mathrm{N}^{\circ} 48$. Washington, D.C.: Banco Mundial; 1995.

16. Drummond MF, Stoddart GI, Torrance GW. Methods for the economic evaluation of health care programmes. New Hamilton: Oxford Medical Publications; 1987.

17. Fisher S, Dornbusch R, Schmalensee R. Economía: métodos de depreciación. $2^{\mathrm{a}}$ ed. Boston: McGraw-Hill, 1990: 418-422.
18. Las Heras JM. Estado eficiente. Sistemas de administración financiera gubernamental Córdoba, Argentina: EUDECOR; 1999.

19. Elio L. Beneficios y beneficiarios: una introducción a la estimación de efectos distributivos en el análisis de costo beneficio. Washington, D.C.: Banco Interamericano de Desarrollo; 1998.

20. Cuervo J, Varela J, Belenes R. Gestión de hospitales-nuevos instrumentos y tendencias. Madrid: Vicens Vives; 1996.

21. Presman G. Los servicios de salud en Argentina. Difusión y uso de la tecnología médica. Buenos Aires: Docencia; 1995.

Manuscrito recibido el 18 de septiembre de 2000. Aceptado para publicación, tras revisión, el 17 de abril de 2001.

\section{APÉNDICE 1. Definiciones del estándar de los acontecimientos indicadores de alta complejidad y de una de las nueve variables}

\section{Definición del estándar de los acontecimientos indicadores de alta} complejidad

- Paciente adulto que realiza tres consultas: consulta externa inicial, consulta preanestésica y consulta preoperatoria cardiológica.

- Internación preoperatoria de $24 \mathrm{~h}$.

- Acto quirúrgico único o múltiple de $4 \mathrm{~h}$ de duración, desde la entrada al quirófano hasta la salida hacia la UCI o UTI, en donde permanece internado $24 \mathrm{~h}$.

- Uso de intensificador de imágenes en quirófano.

- Anestesia: dos peridurales o raquídeas por cada general.

- Internación posoperatoria de 7 días en sala común con curaciones diarias.

- Estudios complementarios: pruebas de laboratorio preoperatorias y posoperatorias rutinarias, ECG preoperatorio y cultivo y antibiograma del drenaje. Radiografía: 10 disparos durante todo el período de internación. Una resonancia magnética.

- Medicamentos: analgésicos (dextropropoxifeno + dipirona) cada $6 \mathrm{~h}$ durante 7 días; antibióticos (gentamicina + cefalosporina) a dosis terapéuticas convencionales durante 5 días; heparinoides, uno por día durante 4 días.

- Transfusión sanguínea: una unidad.

- Descartables quirúrgicos: Steri Drape (NR), Hemosuctor, Dexon (NR) o similar №1 y №0.
- Quinesoterapia: 7 días.

- Implante: precio medio de mercado de una prótesis total de cadera o instrumentación vertebral estándar o clavo endomedular acerrojado.

- Complicaciones: ninguna.

Definición de la variable "quirófano: medicamentos e insumos"

- Anestésicos: costo de todos los medicamentos utilizados para lograr el éxito del procedimiento anestésico, su inducción, mantenimiento y recuperación.

- Soluciones parenterales: costo de soluciones salinas, dextrosadas u otras administradas durante la inducción, mantenimiento y recuperación anestésica y las necesarias para el lavado del campo quirúrgico.

- Descartables: costo de los elementos descartables utilizados durante el acto quirúrgico (agujas, adhesivos, suturas, vendas enyesadas, etc.)

- Otros: costo de expansores plasmáticos, sangre y sus derivados utilizados antes, durante y después del acto quirúrgico. Costo del uso proporcional de los métodos de esterilización en general, amortización de mobiliario, sala de esterilización, uso de los ambientes y del personal necesario, excluido el específico de quirófano. 
ABSTRACT Objective. To determine the operational cost of a hospital's surgical unit, compare those results with standards, and determine the incidence of the costs; to produce an effective methodology for this purpose that can be applied to all kinds of medical units using any low-cost widely available computer database system, with that methodology being utilized for ongoing management control.

Ongoing health management control by cost monitoring through tracer indicators
Methods. This study was carried out in 1999 in the Orthopedics and Traumatology Unit of the "Domingo Funes" Public Hospital, in the province of Córdoba, Argentina. Of the 817 patients who underwent diagnostic or therapeutic procedures in that unit during the year, we selected the 154 of them who were hospitalized and required surgical or instrumental procedures. The study used the tracer event method described by Kessner. Through a consensus process, medical specialists and health economists selected and defined these indicators, which were categorized in relation to their complexity (high, medium, limited, or none). Direct and indirect costs were assessed in order to study their contribution to total cost. Total cost was estimated using nine variables that were applied to the different tracer events.

Results. The largest portion of total cost, both real and standard, was based on the "bed-day" variable. Of this bed-day cost for all tracer events, $51.2 \%$ was due to an indirect cost of "social hospitalization" (keeping patients hospitalized for such reasons as transportation difficulties or delays in receiving requested medical supplies) and $48.8 \%$ to a direct cost of true therapeutic hospitalization. This bed-day cost was $117 \%$ higher for the real costs as compared to the standard costs. The indirect costs of social hospitalization and of therapeutic supplies made up nearly half of the total real cost. Excluding the indirect costs, the real cost of all the tracer events exceeded the standard cost by only $6.4 \%$. Including the indirect costs of social hospitalization and of therapeutic supplies, the real cost exceeded the standard cost by $39.8 \%$. The amount spent on medical staff and support personnel represented just $11.5 \%$ of the total cost.

Discussion. Suggestions are provided in various areas, including solutions for the high cost of social hospitalization and therapeutic supplies, the cost-control benefits of creating protocols for procedures and treatments, and the rational utilization of technology in order to improve efficiency and reduce risks. The wider application of this procedure is recommended for cost-management control in all hospital units carrying out surgery or other medical procedures. 